\title{
Sonografiekurse nach den Richtlinien der ÖGUM
}

\section{Abdomen}

- 25.09.-27.09.2013 Salzburg: Fortgeschrittenenkurs Abdomen

2 20.11.-22.11.2013 Salzburg: Grundkurs Abdomen

Leitung: OA DR. Hollerweger;

kontakt@bhb-sonokurse.at

16.10.-18.10.2013 Baden: Grundkurs Abdomen

Leitung \& Auskunft: Prof. Dr. N. Gritzmann norbert.gritzmann@gmail.com

17.10.-19.10.2013 Hohenems: Abschluß- \& Refresherkurs Abdomen

Leitung \& Auskunft: OA Dr. Gehmacher, sekretariat.interne@lkhh.at

Bewegungsapparat / Nervensystem

- 26.09.-28.09.2013 Wien: 4th Interdisciplinary Musculoskeletal Ultrasound Course Vienna Leitung \& Auskunft: Ass Prof.Prov.Doz.Dr. Claudia Schueller-Weidekamm,

us-course-vienna@meduniwien.ac.at
- 03.10.-05.10.2013 Graz: Musculoskeletal Sonography Course in Rheumatology Leitung \& Auskunft: Dr. Christian Dejaco, noelle.tybery@klinikum-graz.at

- 18.10.-19.10.2013 Baden: Ultraschallspezialkurs Gelenke/Weichteile/ Nerven Leitung \& Auskunft: Prof. Dr. N. Gritzmann; norbert.gritzmann@gmail.com

08.11.-09.11.2013 Innsbruck: 4. Sono- Workshop Innsbruck: Bewegungsapparat - „Die Obere Extremität“"

Leitung \& Auskunft: Univ. Doz.Dr. A. Klauser; andrea.klauser@i-med.ac.at

\section{Echokardiografie}

- 07.11.-09.11.2013 Linz: Pädiatrische Echokardiografie Grundkurs

Leitung \& Auskunft: Prim. Univ.Doz.Dr. G. Tulzer; gerald.tulzer@gespag.at
Gynäkologie / Geburtshilfe

- 29.11.-30.11.2013 Seggau: ErsttrimesterUpdate in Theorie und Praxis Leitung \& Auskunft: OA Dr. Barbara Pertl, marianne.steinwender@lkh-deutschlandsberg.at

Kopf/Hals / Gefässe

- 07.10.-12.10.2013 Graz: Schilddrüsensonografie, Grundkurs

Leitung \& Auskunft: Dr. Wolfgang Buchinger, fortbildung@aekstmk.or.at

\section{Pädiatrie}

- 14.11.- 17.11.2013 Leoben: 22. Steirisches Seminar "Pädiatrische Ultraschalldiagnostik“ Leitung \& Auskunft: OA Dr. Schweintzger; gerolf.schweintzger@lkh-leoben.at

- 07.11.- 09.11.2013 Linz: Grundkurs pädiatrische Echokardiografie Leitung \& Auskunft: Prim. Univ. Doz. Dr. Tulzer,gerald.tulzer@gespag.at 